\title{
Multivariate analysis of lifestyle, constitutive and body composition factors influencing bone health in community-dwelling older adults from Madeira, Portugal
}

\author{
Élvio Rúbio Gouveia ${ }^{\mathrm{a}, *}$, Cameron Joseph Blimkie $^{\mathrm{b}}$, José António Maia ${ }^{\mathrm{c}}$, Carla Lopes ${ }^{\mathrm{d}}$, \\ Bruna Raquel Gouveia ${ }^{\mathrm{e}}$, Duarte Luís Freitas ${ }^{\mathrm{a}}$ \\ ${ }^{a}$ Department of Physical Education and Sports, University of Madeira, Funchal, Portugal \\ ${ }^{\mathrm{b}}$ McMaster University, Department of Kinesiology, Faculty of Science, Hamilton, Ontario, Canada \\ ${ }^{\mathrm{c}}$ CIFI2D and Faculty of Sport, University of Porto, Porto, Portugal \\ ${ }^{\mathrm{d}}$ Department of Clinical Epidemiology, Predictive Medicine and Public Health, Faculty of Medicine and Institute of Public Health, University of Porto, Porto, \\ Portugal \\ ${ }^{\mathrm{e}}$ Health Technologies, Competence Centre, University of Madeira, Funchal, Portugal
}

\section{A R T I C L E IN F O}

\section{Article history:}

Received 7 September 2013

Received in revised form 28 February 2014

Accepted 7 March 2014

Available online 15 March 2014

\section{Keywords:}

Aging

Body composition

Bone mineral density

Physical activity

\begin{abstract}
A B S T R A C T
This study describes the association between habitual physical activity (PA), other lifestyle/constitutive factors, body composition, and bone health/strength in a large sample of older adults from Madeira, Portugal.

This cross-sectional study included 401 males and 401 females aged 60-79 years old. Femoral strength index (FSI) and bone mineral density (BMD) of the whole body, lumbar spine (LS), femoral neck (FN), and total lean tissue mass (TLTM) and total fat mass (TFM) were determined by dual-energy X-ray absorptiometry-DXA. PA was assessed during face-to-face interviews using the Baecke questionnaire and for a sub-sample by Tritrac accelerometer. Demographic and health history information were obtained by telephone interview through questionnaire.

The relationship between habitual PA variables and bone health/strength indicators (whole body BMD, FNBMD, LSBMD, and FSI) investigated using Pearson product-moment correlation coefficient was similar for females $(0.098 \leq r \leq 0.189)$ and males $(0.104 \leq r \leq 0.105)$. Results from standard multiple regression analysis indicated that the primary and most significant predictors for FNBMD in both sexes were age, TLTM, and TFM. For LSBMD, the most significant predictor was TFM in men and TFM, age, and TLTM in females. Our regression model explained $8.3-14.2 \%$ and $14.8-29.6 \%$ of the total variance in LSBMD and FNBMD for males and females, respectively.

This study suggests that habitual PA is minimally but positively associated with BMD and FSI among older adult males and females and that body composition factors like TLTM and TFM are the strongest determinants of BMD and FSI in this population.
\end{abstract}

(c) 2014 Elsevier Ireland Ltd. All rights reserved.

\section{Introduction}

Bone mass, strength and quality in older adults have been associated with genetic factors (Eisman, 1999) and several environmental influences, one of the most important of which is habitual PA (Nguyen, Center, \& Eisman, 2000). Bone-formation

\footnotetext{
* Corresponding author at: Departamento de Educação Física e Desporto, Universidade da Madeira, Campus Universitário da Penteada, 9000-390 Funchal, Portugal. Tel.: +351 291 705313; fax: +351 291705249 .

E-mail addresses: erubiog@uma.pt, rubiogouveia@gmail.com (\&.R. Gouveia).
}

declines with advancing age (Stenderup, Justesen, Clausen, \& Kassem, 2003) as does the levels of habitual PA (Daly et al., 2008); therefore, in both genders, but particularly in women, there is an increased loss of bone mass with aging (Jones, Nguyen, Sambrook, Kelly, \& Eisman 1994). The enhanced bone fragility and consequent increase in fracture risk among the older adults has been linked to changes in intrinsic material properties (i.e. mass, density, stiffness, and strength) and its gross geometric characteristics (size, shape, cortical thickness, cross-sectional area, and trabecular architecture) (Kanis et al., 2008; Khan et al., 2001).

In Europe, the number of all osteoporotic fractures in 2000 was estimated at 3.79 million, of which 0.89 million were hip fractures 
(179 000 hip fractures in men and 711000 in women) (Kanis \& Johnell, 2005). The incidence of hip fractures/year in women and men over 65 years was 67.9/10 000 and 26.1/10 000, respectively (International Osteoporosis Foundation [IOF], 2001). The total direct cost of this disease was estimated at 31.7 billion Euros, which is expected to increase to 76.7 billion Euros in 2050, based on projected demographics in Europe (Kanis \& Johnell, 2005). In Portugal, the number of hip fractures increased from 8500 in 2000 (rate: 8.24 per 10000 ) to 9821 in 2007 (rate: 9.26 per 10000 ); the direct hospital costs were 51321300 Euros and 53433131 Euros, respectively in 2000 and 2007 (IOF, 2008).

Portugal is considered one of the most aged countries of Europe with $17.1 \%$ of its total population older than 65 years of age in 2008 (Statistics Portugal, 2009). With its relatively low fertility rate and rapidly expanding population of older adults, Portugal has been recognized as a high risk country for the development of hip fracture (Kanis \& Johnell, 2005). Particularly in the Portuguese Autonomous Region of Madeira, older adults (65 years and older) are projected to comprise approximately $57.4 \%$ of the total population by 2050. The main occupations in this region are farming and construction work. There are almost as many female (47\%) as male farmers (53\%) in Madeira, with an average age of 64 years (Census 2001) (Statistics Portugal, 2002), the highest of all regions of Portugal.

Numerous lifestyle, constitutive (age, height and body mass (BM)) and body composition factors have been implicated as determinants of bone health in the elderly (Dishaman, Heath, \& Lee, 2013; Khan et al., 2001). Habitual PA, one of the key putative environmental determinants of bone health has been associated with increases in bone mass (Felson, Zhang, Hannan, \& Anderson, 1993; Pluijm et al., 2001) and improvements in muscle mass, muscle strength, balance and bone strength, all of which mitigate falls and fractures among the older adults (United States Department of Health and Human Services [USDHHS], 2004). The evidence in support of the benefits of habitual PA for bone health promotion is so compelling that many physicians and public health officials now recommend increased habitual PA and regular exercise programs regardless of age (USDHHS, 2004).

Besides habitual PA, constitutive factors may also influence bone health status (Daly et al., 2008; Hannan et al., 2000; Lane, 2006). Age-related changes in body composition have been considered potential determinants of bone health/density with aging. Although, their relative importance remains equivocal, changes in BM, TLTM and TFM have each been identified as having important (Dargent-Molina, Poitiers, Bréart, \& EPIDOS Group, 2000; Dytfeld, Ignaszak-Szczepaniak, Gowin, Michalak, \& HorstSikorska, 2011; Felson et al., 1993), independent roles in determining skeletal integrity in older adults (Ho-Pham, Nguyen, Lai, \& Nguyen, 2010). Lean mass is postulated to be a determinant on bone mass because of the structural and functional relationships between muscle and bone (Looker, Melton, Borrud, Shepherd, \& McGowan, 2009). BM and fat mass could exert protective effects on bone by increasing the mechanical loading forces acting on the skeleton during weight-bearing, with fat mass having an additional potential influence through the conversion of steroids to estrogen (Reid, Plank, \& Evans, 1992). Age-related changes in general health and socio-economic status (Booth, Owen, Bauman, Clavisi, \& Leslie, 2000; Lim \& Taylor, 2005), and in smoking, calcium intake, alcohol consumption, nutrition status and prescriptive medications (Felson et al., 1993; Hannan et al., 2000) have also been identified as equivocal yet potential determinants of bone health in older adults.

While fragility fractures may be an inevitable consequence of aging, the morbidity, mortality and financial burden of osteoporosis may be mitigated by management of known risk factors. No attempt has been made in either Portugal or the Autonomous
Region of Madeira (ARM), to identify or characterize relationships between potential determinants of bone health/strength in Portuguese older adults. The purpose of this study was to describe the association between level of habitual PA, other lifestyle and constitutive factors (stature, BM, lean tissue mass, and fat mass) and bone health/strength (assessed as multi-site BMD and FSI) in a large Portuguese sample of active community dwelling older adults men and women (60-79 years), controlling for many of the known important confounding and covariate influences among potential determinants. The Autonomous Region of Madeira represents a relatively unique and isolated geographic and cultural region in Europe. It is uncertain whether the relationships among putative determinants of bone health evident in other more heterogeneous regions of Europe apply also to Madeira. Anticipating high occupational participation rates from the Census data, we hypothesized that constitutive factors rather than habitual PA levels, would be stronger determinants of bone density and strength in this population.

\section{Methods}

\subsection{Study design and participants}

This cross-sectional study included 802 participants (401 males and 401 females) distributed similarly over four age-cohorts (6064, 65-69, 70-74, and 75-79 years). Using GPower 3.1 software (Erdfelder, Faul, \& Buchner, 1996), sample size estimations were done for correlations and regression analysis using Cohen's effect size of 0.25 , alpha $=0.05$ and a power of 0.80 . The suggested sample size varied between 100 and 269 subjects. In total, the sample comprised $2.1 \%$ of the older adults from ARM (60-79 years). Participants were able to walk independently and available to visit our laboratory at the University of Madeira on their own. Proportional regional (geographic) representation was determined by stratified sampling based on Census 2001 data from the Portuguese Statistics National Institute (Statistics Portugal, 2002) with the number of subjects per age cohort and sex serving as stratification factors. This is a relatively unique geographically and culturally isolated group of volunteers recruited via advertisements for a large study on bone health and PA distributed via newspapers and through churches, senior groups and senior centers throughout the ARM in 2008-09.

The study was approved by the University of Madeira, the Regional Secretary of Education and Culture, and the Regional Secretary of Social Affairs. All participants were informed about the nature and purposes of the study and written informed consent was obtained from each subject.

\subsection{Anthropometry and bone densitometry}

BM (kg) was measured with a balance scale accurate to $0.1 \mathrm{~kg}$ (Seca alpha digital scales model 770 , Germany) and standing height $(\mathrm{cm})$ with a Holtain stadiometer (Holtain Ltd., Crymych, United Kingdom) accurate to $0.1 \mathrm{~cm}$. Subjects wore light, indoor clothing without shoes during the measurements.

BMD $\left(\mathrm{g} / \mathrm{cm}^{2}\right)$ was determined by dual-energy X-ray absorptiometry-DXA (Lunar Prodigy Primo, with technologic fan beam - GE Healthcare, Encore 2007 software version 11.40.004). After removing all objects suspected or known to contain metal, participants were positioned by the technician according to the manufacturer's recommended protocol. Subjects were in a supine position and the following sites were investigated: whole body, LS (anterior-posterior), and hip (FN, trochanter, Ward's triangle and total hip). Furthermore, the scans yielded information on body composition, including TLTM and TFM. 
In addition to the conventional densitometry measurements, structural variables were also determined using the Hip Strength Analysis program, including hip axis length and cross-sectional moment of inertia (CSMI). These bone geometry variables were used to calculate the FSI, the ratio of estimated compressive yield strength of the FN to the expected compressive stress of a fall on the greater trochanter adjusted for each subject's age, height and BM (Yoshikawa et al., 1994). FSI provides an estimate of functional bone strength of the hip region based on both its level of mineralization and the geometric distribution of mineral around central axis of the FN (Beck, Ruff, Warden, Scott, \& Rao, 1990).

Scans were standardized daily against a calibration phantom; the precision error expressed as the coefficient of variation (CV\%) was $0.31 \%$. Scans were taken alternately by four different technicians over the course of data collection. All technicians received an identical 5 days DXA training course before the start the study using the manufacturer's recommended protocol. Reliability of our DXA measurements was determined on a subsample of 17 males and females aged $69.3 \pm 5$ years. Technicians were paired and members of each pair performed separate LS and hip scans on half the subjects each ( 9 and 8 subjects, respectively, per pair). Subjects were repositioned after every scan. Results from both pairs of assessors were pooled and the technical error of the measurements (TEM) was determined. TEM was used to determine inter-observer error, as occurs when two technicians independently measure the same thing. The values ranged from $0.19 \%$ for total hip to $0.50 \%$ for the LS. Inter-observer reliability was also determined using the CV. The CV\% was $1.72 \%$ for LS, $2.10 \%$ for the FN, $2.53 \%$ for Ward's triangle and $0.88 \%$ for the total hip.

\subsection{PA measures}

Habitual PA was assessed during face-to-face interviews using the Baecke questionnaire developed in the Netherlands (Baecke, Burema, \& Frijters, 1982), with a reference time period of last year. This questionnaire includes a total of 16 questions classified into three specific domains: PA at work/housework, sport and leisure time, the latter excluding sports. If the subjects were not employed or if they were retired, their occupation was coded as homemaker. The questionnaire also provides a measure of habitual PA which is the sum of these three specific domains. Numerical coding for most response categories varied from 1 to 5 (Likert scale) ranging from never to always or very often. A detailed description of the scoring procedures for calculation of habitual PA and its subcomponent categories (PA at work, sport and leisure time) is provided by Baecke et al. (1982). The questionnaire took about $20 \mathrm{~min}$ to complete.

Interviews were taken alternately by four different researchers over the course of data collection. Previously, intra-class correlation-coefficients were calculated to determine the test-retest reliability of the questionnaire in a pilot study involving 32 males and 59 females $(68.3 \pm 7.6$ years). Over an interval of 1 week, correlations ranged between $0.83,0.85$ and 0.85 for the work, sport and leisure-time indices, respectively. Our reliability scores for work and sport PA were similar to those obtained by Baecke et al. (1982) in a sample of Dutch adult men and women $(0.88,0.81)$ and with a more recent study by Ono et al. (2007) of 61 middle aged (53.3 years) women $(0.84,0.83)$. However, our correlations were higher for leisure time index than those reported by either Baecke et al. (1982) or Ono et al. (2007), 0.74 and 0.78 , respectively. The validity of the Baecke questionnaire has also been established by Ono et al. (2007) for this population against the more objective measure of movement counts using digital pedometry and uniaxial accelerometry (Lifecorder, Suzuken Co., Nagoya, Japan); correlations ranged from 0.30 to 0.49 for the 3 dimensions of habitual PA assessed with the Baecke questionnaire in this study.
As an internal validation of the Baecke Questionnaire approach, of the relationships between habitual levels of PA and bone health indicators, in our study PA was also assessed using the RT3 triaxial accelerometer in a sub-sample of 173 older adults, 89 males and 84 females, age 60-79 years. The RT3 accelerometer measures acceleration in each anatomical axis with vertical $(x)$, anterioposterior $(y)$, and mediolateral $(z)$ measurements. To determine the total daily PA "counts", we used the square-root of the sum of squared accelerations for each axis, which provides the vector magnitude (VM) in counts per minute (counts/min). Participants were instructed to wear the RT3 for a 7-day period during waking hours and remove it for sleeping, swimming and bathing only.

For inclusion in the present study, participants were required to have worn the accelerometer on at least five complete days (including Saturday and Sunday). Participants were instructed to wear the RT3 for a 7-day period during waking hours and remove it for sleeping, swimming and bathing only. For inclusion in the present study, participants were required to have worn the accelerometer on at least five complete days (including Saturday and Sunday). The cross correlations were generally weak, when the relationships between the more objective measure of PA and the key dependent variables were examined in the regression analysis, PA was persistently still a relatively weak determinant of bone sites and FSI in this population $(0.002 \leq \beta \leq 0.098$ and $0.042 \leq \beta \leq 0.084$ for men and women, respectively). We also correlated the total score of PA from Baecke questionnaire with the total "counts" from RT3 using the Pearson product-moment correlation coefficient. However, the correlations for men and women together were positive, but non-significant $(r=0.131$; $p>0.87$ )

\subsection{Health questionnaire and dietary intake}

Demographic information and a complete health history were obtained by telephone interview. The health questionnaire employed in the FallProof! Program (Rose, 2003) was used to assess smoking history and medication.

Dietary intake was estimated using a semi-quantitative food frequency questionnaire developed by the Department of Hygiene and Epidemiology of Porto University Medical School, and previously validated in 2415 Portuguese participants (926 males and 1489 females) aged 18-92 years (Lopes, 2000). The semiquantitative food frequency questionnaire was done with a reference time period of last year. This questionnaire was used to quantify calcium and alcohol using the software Food Processor Plus ${ }^{\circledR}$ (ESHA Research, Salem-Oregon, 1997), adapted to Portuguese traditional foods and dishes.

\subsection{Statistical analysis}

Descriptive characteristics of participants were reported as means \pm SDs. All data were tested for normality by the KolmogorovSmirnov statistic. When required, non-normal distributed characteristics were appropriately transformed using $\log 10$, square root or inverse transform functions.

Sex specific bivariate associations between the bone health indicators (BMD and FSI) and putative predictors of bone health (age, BM, TLTM, total calcium intake, alcohol consumption and habitual PA in women and sports related PA in men) were calculated for all age-cohorts combined using Pearson correlations. Sex-specific standard multiple regression analysis was then used to identify the independent contribution of the individual and combined predictors for BMD at the different skeletal sites. Betas, namely standardized regression coefficients, were used to assess the relative independent contributions of each predictor or combination of predictors, and the adjusted $R^{2} \mathrm{~s}$ indicated the 
Table 1

Age and sex-specific descriptive characteristics.

\begin{tabular}{|c|c|c|c|c|c|c|}
\hline & \multicolumn{4}{|c|}{ Age groups (years) } & \multirow[t]{3}{*}{$p$ value } & \multirow[t]{3}{*}{ Comparison } \\
\hline & $60-64(1)$ & $65-69(2)$ & $70-74(3)$ & $75-79(4)$ & & \\
\hline & Mean \pm SD & Mean \pm SD & Mean \pm SD & Mean \pm SD & & \\
\hline Men & $(n=103)$ & $(n=99)$ & $(n=107)$ & $(n=92)$ & & \\
\hline Height $(\mathrm{cm})$ & $166.9 \pm 5.2$ & $165.9 \pm 6.2$ & $164.5 \pm 6.1$ & $164.6 \pm 6.2$ & 0.011 & $1,2>3,4$ \\
\hline $\mathrm{BM}(\mathrm{kg})$ & $80.3 \pm 12.1$ & $79.7 \pm 13.1$ & $79.9 \pm 13.3$ & $75.8 \pm 13.0$ & 0.058 & - \\
\hline TLTM (kg) & $54.3 \pm 5.9$ & $53.3 \pm 5.8$ & $52.8 \pm 5.5$ & $51.2 \pm 6.3$ & 0.003 & $4<3,2,1$ \\
\hline TFM (kg) & $22.6 \pm 8.1$ & $23.3 \pm 7.9$ & $23.6 \pm 8.6$ & $21.5 \pm 8.2$ & 0.285 & \\
\hline PA work (1-5 units) & $2.8 \pm 0.6$ & $2.6 \pm 0.5$ & $2.6 \pm 0.6$ & $2.7 \pm 0.5$ & 0.066 & \\
\hline PA leisure (1-5 units) & $2.6 \pm 0.6$ & $2.5 \pm 0.6$ & $2.5 \pm 0.7$ & $2.5 \pm 0.5$ & 0.520 & \\
\hline PA sport (1-5 units) & $2.2 \pm 0.6$ & $2.2 \pm 0.6$ & $2.0 \pm 0.6$ & $1.9 \pm 0.5$ & 0.015 & $4<2,1$ \\
\hline Habitual PA (3-15 units) & $7.6 \pm 1.3$ & $7.3 \pm 1.2$ & $7.1 \pm 1.4$ & $7.2 \pm 1.2$ & 0.065 & - \\
\hline Diet. calcium (mg/day) & $721.7 \pm 321.7$ & $743.7 \pm 365.2$ & $723.9 \pm 350.6$ & $734.5 \pm 384.9$ & 0.083 & - \\
\hline Alcohol (dl/day) & $16.7 \pm 20.5$ & $13.1 \pm 17.3$ & $11.6 \pm 18.2$ & $9.2 \pm 15.3$ & 0.028 & $4<3,2,1$ \\
\hline Women & $(n=102)$ & $(n=108)$ & $(n=98)$ & $(n=93)$ & & \\
\hline Height $(\mathrm{cm})$ & $154.2 \pm 5.4$ & $153.8 \pm 5.5$ & $152.0 \pm 5.8$ & $150.1 \pm 5.4$ & $<0.001$ & $4<2,1 ; 3<1$ \\
\hline $\mathrm{BM}(\mathrm{kg})$ & $72.2 \pm 11.7$ & $71.2 \pm 12.7$ & $70.6 \pm 10.6$ & $67.8 \pm 11.5$ & 0.063 & $4<3,2,1$ \\
\hline TLTM (kg) & $39.9 \pm 4.9$ & $40.0 \pm 5.5$ & $39.3 \pm 3.9$ & $38.2 \pm 4.3$ & 0.033 & $4<2$ \\
\hline TFM (kg) & $29.7 \pm 7.8$ & $28.7 \pm 8.0$ & $28.2 \pm 7.3$ & $27.1 \pm 7.8$ & 0.131 & - \\
\hline PA work (1-5) & $2.8 \pm 0.6$ & $2.8 \pm 0.4$ & $2.7 \pm 0.4$ & $2.5 \pm 0.4$ & $<0.001$ & $4<2,1$ \\
\hline PA leisure (1-5) & $2.5 \pm 0.7$ & $2.5 \pm 0.5$ & $2.4 \pm 0.5$ & $2.4 \pm 0.6$ & 0.071 & - \\
\hline PA sport $(1-5)$ & $2.2 \pm 0.6$ & $2.3 \pm 0.6$ & $2.3 \pm 0.6$ & $2.1 \pm 0.6$ & 0.072 & - \\
\hline Habitual PA (3-15) & $7.5 \pm 1.3$ & $7.5 \pm 1.1$ & $7.3 \pm 1.1$ & $6.9 \pm 1.2$ & 0.002 & $4<2,1$ \\
\hline Diet. calcium (mg/day) & $906.5 \pm 387.3$ & $817.9 \pm 381.2$ & $821.8 \pm 398.6$ & $796.5 \pm 384.5$ & 0.197 & - \\
\hline Alcohol (dl/day) & $2.1 \pm 3.9$ & $1.5 \pm 4.1$ & $1.2 \pm 2.7$ & $1.3 \pm 2.9$ & 0.178 & - \\
\hline
\end{tabular}

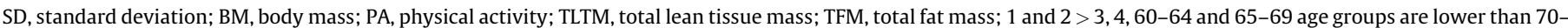

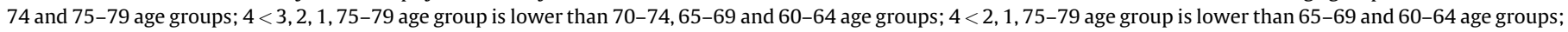
$3<1,70-74$ age group is lower than $60-64$ age group; $4<2,75-79$ age group is lower than $65-69$ age group.

percentage of explained variance in the bone health outcomes. The level of significance was set at $p<0.05$. All analyses were performed using SPSS (version 18.0).

\section{Results}

Table 1 shows that age-related declines were significant $(p<0.05)$ for height and TLTM for both males and females. BM declined significantly across age only in women and TFM was unchanged across age in males and females.

Likewise, sports related PA for men, work related PA and habitual PA for women, decreased significantly across age-groups by $13.6 \%, 10.7 \%$, and $8.0 \%$, respectively. There were no significant age related declines for any of the other habitual PA variables for men and women. Only men demonstrated a significant age-related reduction in alcohol consumption and there were no significant age-related differences in calcium intake for either sex.
BMD for all sites and for both sexes was, with one exception (LS males 65-69 years), highest in the youngest age-cohort and for the most part decreased progressively with advancing age-cohort (Table 2) for both sexes. BMD declined significantly (9.6-21.1\%) with age (60-79 years) at all sites for women whereas significant age-reductions were observed only for the FN (9.8\%) for males. FSI was highest in the youngest age-cohort in both sexes, did not change significantly across ages for males, but decreased progressively and significantly with increasing age-cohort in females.

With the exception of the LS for males, BMD at multiple sites were significantly negatively correlated with age in both genders (Table 3). All BMD measures were significantly positively correlated with height, BM, TLTM and TFM for both males and females. Sport related PA (Baecke Questionnaire) was the only activity category to relate significantly with BMD for males, correlating weakly, but nonetheless positively, with LS BMD. The

Table 2

Age and sex-specific descriptive characteristics for BMD and FSI.

\begin{tabular}{|c|c|c|c|c|c|c|}
\hline & \multicolumn{4}{|c|}{ Age groups (years) } & \multirow[t]{3}{*}{$p$ value } & \multirow[t]{3}{*}{ Comparison } \\
\hline & $60-64(1)$ & $65-69(2)$ & $70-74(3)$ & $75-79(4)$ & & \\
\hline & Mean \pm SD & Mean \pm SD & Mean \pm SD & Mean \pm SD & & \\
\hline \multicolumn{7}{|l|}{ Men } \\
\hline Total body BMD $\left(\mathrm{g} / \mathrm{cm}^{2}\right)$ & $1.23 \pm 0.10$ & $1.22 \pm 0.11$ & $1.22 \pm 0.11$ & $1.19 \pm 0.09$ & 0.077 & - \\
\hline $\operatorname{LS} \operatorname{BMD}\left(\mathrm{g} / \mathrm{cm}^{2}\right)$ & $1.19 \pm 0.18$ & $1.21 \pm 0.21$ & $1.18 \pm 0.24$ & $1.14 \pm 0.19$ & 0.276 & - \\
\hline FN BMD $\left(\mathrm{g} / \mathrm{cm}^{2}\right)$ & $1.02 \pm 0.15$ & $0.99 \pm 0.14$ & $0.95 \pm 0.14$ & $0.92 \pm 0.13$ & $<0.001$ & $4<2,1 ; 3<1$ \\
\hline FSI & $1.86 \pm 0.45$ & $1.74 \pm 0.52$ & $1.76 \pm 0.51$ & $1.83 \pm 0.57$ & 0.264 & - \\
\hline \multicolumn{7}{|l|}{ Women } \\
\hline Total body BMD $\left(\mathrm{g} / \mathrm{cm}^{2}\right)$ & $1.14 \pm 0.09$ & $1.10 \pm 0.10$ & $1.07 \pm 0.09$ & $1.03 \pm 0.08$ & $<0.001$ & $4<3,2,1 ; 3$ and $2<1$ \\
\hline LS BMD (g/cm²) & $1.06 \pm 0.18$ & $1.01 \pm 0.16$ & $0.96 \pm 0.18$ & $0.95 \pm 0.16$ & $<0.001$ & $1>2,3,4$ \\
\hline FN BMD $\left(\mathrm{g} / \mathrm{cm}^{2}\right)$ & $0.92 \pm 0.13$ & $0.88 \pm 0.12$ & $0.83 \pm 0.10$ & $0.78 \pm 0.10$ & $<0.001$ & $4<3,2,1 ; 3<2,1 ; 2<1$ \\
\hline FSI & $1.61 \pm 0.47$ & $1.53 \pm 0.39$ & $1.48 \pm 0.38$ & $1.44 \pm 0.37$ & 0.027 & $1>2,3,4$ \\
\hline
\end{tabular}

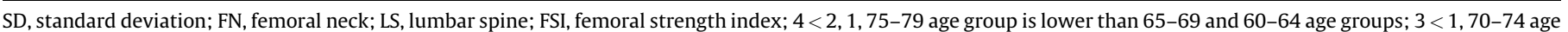

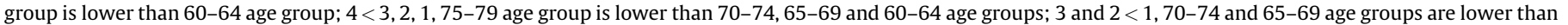

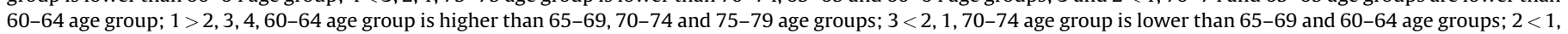
65-69 age group is lower than 60-64 age group. 
Table 3

Sex-specific Pearson correlations between BMD indicators, FSI and selected descriptive characteristics.

\begin{tabular}{|c|c|c|c|c|}
\hline Characteristics & Total body BMD $\left(\mathrm{g} / \mathrm{cm}^{2}\right)$ & LS BMD $\left(\mathrm{g} / \mathrm{cm}^{2}\right)$ & FN BMD $\left(\mathrm{g} / \mathrm{cm}^{2}\right)$ & FSI \\
\hline \multicolumn{5}{|l|}{ Men } \\
\hline Age (years) & $-0.110^{\ddagger}$ & - & $-0.259^{\dagger}$ & - \\
\hline Height $(\mathrm{cm})$ & $0.279^{\dagger}$ & $0.158^{\dagger}$ & $0.262^{\dagger}$ & $-0.147^{\dagger}$ \\
\hline $\mathrm{BM}(\mathrm{kg})$ & $0.425^{\dagger}$ & $0.293^{\dagger}$ & $0.309^{\dagger}$ & $-0.343^{\dagger}$ \\
\hline TLTM (kg) & $0.389^{\dagger}$ & $0.179^{\dagger}$ & $0.308^{\dagger}$ & $-0.165^{\dagger}$ \\
\hline TFM (kg) & $0.319^{\dagger}$ & $0.292^{\dagger}$ & $0.228^{\dagger}$ & $-0.383^{\dagger}$ \\
\hline PA work (1-5 units) & - & - & - & $0.105^{\ddagger}$ \\
\hline PA leisure (1-5 units) & - & - & - & - \\
\hline PA sport (1-5 units) & - & $0.104^{\ddagger}$ & - & - \\
\hline Habitual PA (3-15 units) & - & - & - & - \\
\hline Diet. calcium (mg/day) & - & - & - & - \\
\hline Smoking (units) & $-0.147^{\ddagger}$ & $-0.124^{\ddagger}$ & - & - \\
\hline Alcohol (dl/day) & - & - & - & - \\
\hline Medication (units) & - & $0.123^{\ddagger}$ & - & - \\
\hline \multicolumn{5}{|l|}{ Women } \\
\hline Age (years) & $-0.394^{\dagger}$ & $-0.248^{\dagger}$ & $-0.443^{\dagger}$ & $-0.164^{\dagger}$ \\
\hline Height $(\mathrm{cm})$ & $0.396^{\dagger}$ & $0.314^{\dagger}$ & $0.426^{\dagger}$ & - \\
\hline $\mathrm{BM}(\mathrm{kg})$ & $0.530^{\dagger}$ & $0.395^{\dagger}$ & $0.352^{\dagger}$ & $-0.334^{\dagger}$ \\
\hline TLTM (kg) & $0.477^{\dagger}$ & $0.323^{\dagger}$ & $0.336^{\dagger}$ & $-0.220^{\dagger}$ \\
\hline TFM (kg) & $0.486^{\dagger}$ & $0.334^{\dagger}$ & $0.314^{\dagger}$ & $-0.326^{\dagger}$ \\
\hline PA work (1-5 units) & $0.098^{\ddagger}$ & - & $0.164^{\dagger}$ & 0.099 \\
\hline PA leisure (1-5 units) & $0.127^{\ddagger}$ & - & $0.100^{\ddagger}$ & $0.108 \ddagger$ \\
\hline PA sport (1-5 units) & - & - & $0.140^{\dagger}$ & - \\
\hline Habitual PA (3-15 units) & $0.117^{\ddagger}$ & - & $0.189^{\dagger}$ & - \\
\hline Diet. calcium (mg/day) & $0.108^{\ddagger}$ & - & - & $0.108^{\ddagger}$ \\
\hline Smoking (units) & $0.150^{\dagger}$ & $0.150^{\dagger}$ & $0.192^{\dagger}$ & - \\
\hline Alcohol (dl/day) & - & - & - & - \\
\hline Medication (units) & - & - & - & $0.115^{\ddagger}$ \\
\hline
\end{tabular}

FN, femoral neck; LS, lumbar spine; FSI, femoral strength index; BM, body mass; PA, physical activity; TLTM, total lean tissue mass; TFM, total fat mass. Only correlations that were statistically significant were included.

$¥$ Correlation is significant at the 0.05 level (2-tailed).

$\dagger$ Correlation is significant at the 0.01 level (2-tailed).

relationship between habitual PA and BMD in females was generally more positive than for males, with several significant, but similarly weak correlations between habitual PA variables and the various measures of BMD (Table 3). FSI was significantly negatively correlated with age in females but not males, with height in males but not females, and with BM, TLTM and TFM in both sexes. FSI was significantly negatively correlated with work related PA, leisure time PA and dietary calcium intake in females only.

Results from multiple regression analysis modeling containing TLTM, TFM, habitual PA, age and total calcium intake are presented in Table 4 for the FN, LS and FSI measures only. Similar modeling was done for the remaining bone measures but these findings are not reported in these tables. Age, TLTM, TFM for FNBMD in both sexes, TFM in men and TFM, age and TLTM in female for LSBMD, entered as the primary and most significant contributors. Habitual PA contributed significantly to variation in FNBMD in females. In men, habitual PA did not significantly contribute to the variation in BMD at any bone site. Also, dietary calcium intake did not contribute to the variation in BMD of FN or LS in either sex. Our regression model explained 14.2 and $29.6 \%$ of the total variance in the FNBMD in males and females, respectively. For LSBMD, the total variance explained by the model was $8.3 \%$ in males and $14.8 \%$ in females. The regression model explained 13.7 and $14.6 \%$ of the total variation in mechanical FSI in males and females, respectively. In both sexes, age was the most significant predictor for FNBMD, and TFM for LSBMD and for FSI.

\section{Discussion}

The bivariate correlation and multiple regression analyses revealed only minor associations between habitual PA and BMD. Our study reinforces that constitutive and body composition factors are the strongest predictors of BMD and FSI in older males and females. Moreover, associations tended to be sex- and sitespecific.

Additional information about FSI, a derived measure that provides an estimate of hip fracture risk, was also assessed in our study. FSI is not only dependent on femoral BMD, but it is also a function of the spatial distribution of bone mass and bone's intrinsic structural geometric properties such as its diameter, area, length, and angle at the FN. FSI has been recognized as a significant independent predictor of hip fracture risk (Beck et al., 1990; Faulkner et al., 2006). In this study habitual PA was not a significant predictor of FSI in our multiple regression analysis. However, work related PA was significantly correlated with FSI in males and females, whereas the association with leisure time PA was significant only in females. As with our findings for BMD, these results also suggest that PA in general, is only weakly-moderately associated with FSI in older adult men and women of our study.

The association between habitual PA and BMD in older adults has not been extensively studied and collectively the findings are equivocal at best. Uncertainly in this area stems from the fact that previous activity assessment approaches have not fully considered the entire array of putative mechanical loading parameters (type, intensity, frequency and duration of activities) now known to independently or interactively affect skeletal adaptation (Maïmoun \& Sultan, 2011). It is widely acknowledged that bone tissue (cortical bone and porous trabecular bone) adapts its shape and structure according to its mechanical environment. The bone remodeling mechanism is complex and is determined also by a speculative network of interactions influenced by factors such as genetics, growth factors, gender, age, soft tissue composition (TLTM, TFM), lifestyle choices (smoking, alcohol intake), medication, hormones, and nutrition (Khan et al., 2001).

Our findings for BMD are in partial agreement with previous select cross-sectional and longitudinal studies of similarly aged populations. Hannan et al. (2000) and Stewart et al. (2005) failed to 
Table 4

Standard MLR between FN, LS and FSI and putative predictors (TLTM, TFM, habitual PA and total calcium intake).

\begin{tabular}{|c|c|c|c|c|c|}
\hline Predictors & Crude $B \pm$ std. error & Adjusted $B \pm$ std. error & Beta $^{\dagger}$ & $p$ value & $95 \% \mathrm{Cl}^{\mathrm{a}}$ \\
\hline \multicolumn{6}{|l|}{ FN BMD $\left(\mathrm{g} / \mathrm{cm}^{2}\right)$} \\
\hline TLTM $(\mathrm{kg})$ & $0.007 \pm 0.001$ & $0.005 \pm 0.001$ & 0.210 & $<0.001$ & $0.003 ; 0.008$ \\
\hline TFM (kg) & $0.004 \pm 0.001$ & $0.002 \pm 0.001$ & 0.130 & 0.016 & $0.000 ; 0.004$ \\
\hline Habitual PA (5-15 units) & $0.004 \pm 0.006$ & $0.003 \pm 0.005$ & 0.028 & 0.551 & $-0.007 ; 0.014$ \\
\hline Age (years) & $-0.007 \pm 0.001$ & $-0.005 \pm 0.001$ & -0.211 & $<0.001$ & $-0.008 ;-0.003$ \\
\hline Diet. calcium (mg/day) & $2.386^{-5} \pm 0.000$ & $2.326^{-5} \pm 0.000$ & 0.057 & 0.220 & $0.000 ; 0.000$ \\
\hline \multicolumn{6}{|l|}{ Women $\left(R^{2}{ }_{a d j}=0.296\right)$} \\
\hline TLTM (kg) & $0.009 \pm 0.001$ & $0.004 \pm 0.001$ & 0.162 & 0.002 & $0.002 ; 0.007$ \\
\hline TFM (kg) & $0.005 \pm 0.001$ & $0.003 \pm 0.001$ & 0.182 & $<0.001$ & $0.001 ; 0.005$ \\
\hline Habitual PA (units) & $0.020 \pm 0.005$ & $0.013 \pm 0.005$ & 0.124 & 0.004 & $0.004 ; 0.022$ \\
\hline Age (years) & $-0.010 \pm 0.001$ & $-0.008 \pm 0.001$ & -0.369 & $<0.001$ & $-0.010 ;-0.006$ \\
\hline Diet. calcium (mg/day) & $4.037^{-5} \pm 0.001$ & $1.585^{-5} \pm 0.000$ & 0.049 & 0.253 & $0.000 ; 0.000$ \\
\hline \multicolumn{5}{|l|}{ LS BMD $\left(\mathbf{g} / \mathbf{c m}^{2}\right)$} & $\operatorname{Men}\left(R^{2}{ }_{a d j}=0.083\right)$ \\
\hline TLTM $(\mathrm{kg})$ & $0.006 \pm 0.002$ & $0.001 \pm 0.002$ & 0.041 & 0.460 & $-0.002 ; 0.005$ \\
\hline TFM (kg) & $0.008 \pm 0.001$ & $0.007 \pm 0.001$ & 0.282 & $<0.001$ & $0.005 ; 0.010$ \\
\hline Habitual PA (5-15 units) & $0.001 \pm 0.008$ & $0.008 \pm 0.008$ & 0.049 & 0.319 & $-0.008 ; 0.025$ \\
\hline Age (years) & $-0.003 \pm 0.002$ & $-0.002 \pm 0.002$ & -0.044 & 0.374 & $-0.005 ; 0.002$ \\
\hline Diet. calcium (mg/day) & $1.880^{-5} \pm 0.000$ & $2.645^{-5} \pm 0.000$ & 0.043 & 0.369 & $0.000 ; 0.000$ \\
\hline \multicolumn{6}{|l|}{ Women $\left(R^{2}{ }_{a d j}=0.148\right)$} \\
\hline TLTM (kg) & $0.011 \pm 0.002$ & $0.005 \pm 0.002$ & 0.118 & 0.042 & $0.000 ; 0.009$ \\
\hline TFM (kg) & $0.008 \pm 0.001$ & $0.006 \pm 0.001$ & 0.244 & $<0.001$ & $0.003 ; 0.008$ \\
\hline Habitual PA (5-15 units) & $0.006 \pm 0.008$ & $0.003 \pm 0.007$ & 0.018 & 0.704 & $-0.012 ; 0.017$ \\
\hline Age (years) & $0.007 \pm 0.002$ & $-0.006 \pm 0.002$ & -0.176 & $<0.001$ & $-0.009 ;-0.003$ \\
\hline Diet. calcium (mg/day) & $3.267^{-5} \pm 0.000$ & $1.304^{-5} \pm 0.000$ & 0.028 & 0.558 & $0.000 ; 0.000$ \\
\hline \multicolumn{6}{|l|}{ FSI } \\
\hline \multicolumn{6}{|l|}{$\operatorname{Men}\left(R^{2}{ }_{a d j}=0.137\right)$} \\
\hline TLTM $(\mathrm{kg})$ & $-0.014 \pm 0.004$ & $0.000 \pm 0.005$ & 0.005 & 0.931 & $-0.009 ; 0.009$ \\
\hline TFM (kg) & $-0.024 \pm 0.003$ & $-0.024 \pm 0.003$ & -0.383 & $<0.001$ & $-0.031 ;-0.018$ \\
\hline Habitual PA (5-15 units) & $0.030 \pm 0.020$ & $0.005 \pm 0.019$ & 0.012 & 0.803 & $-0.033 ; 0.043$ \\
\hline Age (years) & $-0.002 \pm 0.005$ & $-0.002 \pm 0.004$ & -0.025 & 0.600 & $-0.011 ; 0.006$ \\
\hline Diet. calcium (mg/day) & $5.725^{-5} \pm 0.000$ & $3.820^{-5} \pm 0.000$ & 0.027 & 0.570 & $0.000 ; 0.000$ \\
\hline \multicolumn{6}{|l|}{ Women $\left(R^{2}{ }_{a d j}=0.146\right)$} \\
\hline TLTM (kg) & $-0.019 \pm 0.004$ & $-0.007 \pm 0.005$ & -0.085 & 0.141 & $-0.017 ; 0.002$ \\
\hline TFM (kg) & $-0.017 \pm 0.003$ & $-0.016 \pm 0.003$ & -0.301 & $<0.001$ & $-0.022 ;-0.010$ \\
\hline Habitual PA (5-15 units) & $0.033 \pm 0.017$ & $0.014 \pm 0.016$ & 0.041 & 0.392 & $-0.018 ; 0.046$ \\
\hline Age (years) & $-0.012 \pm 0.004$ & $-0.015 \pm 0.003$ & -0.203 & $<0.001$ & $-0.021 ;-0.008$ \\
\hline Diet. calcium (mg/day) & $6.106^{-5} \pm 0.000$ & $4.959^{-5} \pm 0.000$ & 0.047 & 0.315 & $0.000 ; 0.000$ \\
\hline
\end{tabular}

TLTM, total lean tissue mass; TFM, total fat mass; PA, physical activity.

a $95.0 \%$ confidence interval for $B$-values.

$\dagger$ Significant contribution by an independent variable to the total explained variation in the model $(p<0.05)$.

find any association between PA score and BMD change in older adults males within the age range of our study, whereas Kenny, Prestwood, Marcello, and Raisz (2000) and Nguyen et al. (2000) found a significant positive relationship between PA and FN, but not LSBMD in similarly aged males. A few additional studies, reported positive associations between sport related PA with FN and hip BMD in older adults males (Pluijm et al., 2001; Vuillemin, Guillemin, Jouanny, Denis, \& Jeandel, 2001), and positive associations between PA and BMD using simple univariate analyses, which became weaker or non-significant with more stringent multivariate approaches that adjusted for putative BMD covariates (Lau et al., 2006).

There is more abundant comparative research for older adult women than men, but the results are no less equivocal. Previous studies (Nguyen, Sambrook, \& Eisman, 1998) have shown a modest favorable effect of PA on the rate of bone loss at the FN and significant positive associations with FN but not LSBMD in older adults females (Hagberg et al., 2001; Nguyen et al., 2000; Pluijm et al., 2001). Other kind of analysis provided by Mavroeidi, Stewart, Reid, and Macdonald (2009) have reported significant interactions between PA tertiles, classified according to metabolic (energy expended in carrying out activities expressed in MET h/week) and mechanical components of PA (from ground reaction forces on the skeleton expressed in peak scores), and left and right hip BMD in a large population of older women living in the community. Others have reported no relationship between PA level and BMD in older adults females (Gába, Kapuš, Pelclová, \& Riegerová, 2012; Nahas, Kawakami, Nahas-Neto, Buttros Dde, \& Cangussu, 2011) or insignificant relationships with proxy measures of habitual PA after adjustment for covariates (Schoffl et al., 2008), whereas some have reported specific positive associations with walking (Pluijm et al., 2001) and sports related PA (Vuillemin et al., 2001).

There are few published reports of the association between geometric or derived biomechanical and functional (e.g. bone strength index) measures of bone strength and PA in older adults populations. However, similar to our findings, Nurzenski et al. (2007) and Uusi-Rasi, Sievänen, Pasanen, Beck, and Kannus (2008) reported significant positive associations between aspects of femoral bone geometry and PA in older adults females, whereas there is only one report to our knowledge (Semanick et al., 2005), which likewise, indicated a weak positive relationship between walking and FN section modulus in older adults men.

In our study, the indices of habitual PA were measured by the Baecke questionnaire, which includes three reliable dimensions: PA at work (participants who were retired we classified in low level for occupation), sport during leisure-time, and other PA during 
leisure-time (Baecke et al., 1982). This instrument was also used in slightly younger healthy 57 year old post-menopausal women reported in the study by Walsh, Hunter, and Livingstone (2006) and younger healthy adult Belgians 30-40 years of age (Philippaerts \& Lefevre, 1998). Although our sample is older than those in these reference studies, we trust that the Baecke questionnaire is still a useful instrument since our older population was very active for their age, in relation to others regions from Portugal as reported in Census 2001 (Statistics Portugal, 2002).

Notwithstanding this information, discrepancies among findings regarding PA may be attributed to a variety of factors. Studies often include different measures of PA and its sub-classifications, different age ranges, differing cut points defining age-specific cohorts, variable health status and levels of social independence and co-morbidities, different degrees of statistical sophistication in data analysis, differing levels of historical and habitual PA and variable measures of bone health. Apart these limitations, our findings and the cumulative literature to date suggest that "current" levels of PA contribute only minimally in explaining variation in BMD and FSI among older adults males, with perhaps a slightly stronger influence among older adults females and for certain geometric measures reflecting bone strength in both sexes. Our findings regarding PA were not altogether unexpected, as we anticipated, based on the National Survey (Census 2001) (Statistics Portugal, 2002), a relatively high level of occupational engagement among our subjects, which we hypothesized would reduce variability in PA levels, thereby accentuating broader differences in constitutive factors. In our study, however, only $35.2 \%$ of the men and $11.5 \%$ of the women were active in farming. This differs markedly from the percentage found in the National Census, suggesting that subjects in our study were less occupationally engaged than the regional population at large. Notwithstanding this difference in occupational engagement, our findings nevertheless suggest reduced variability in general levels of PA among our subjects compared to constitutive factors.

In the present study, as hypothesized, constitutive factors like age, height and BM, and body composition factors like TLTM and TFM were consistently more strongly correlated with BMD and FSI than any of our measures of PA. The multiple regression analyses, revealed that these constitutive and body composition factors make the strongest contribution to explained the dependents variables, when the variance explained by all other variables in the model was controlled for. Specifically, age, TLTM and TFM for FNBMD in both genders and TFM in men and TFM and age in women for LSBMD and FSI, Other studies have shown that age (Hannan et al., 2000), BM (Dargent-Molina et al., 2000; Felson et al., 1993), TLTM (Dytfeld et al., 2011; Ho-Pham et al., 2010; Travison, Araujo, Esche, Beck, \& McKinlay 2008) and TFM (Ho-Pham et al., 2010) are important predictors of BMD in older adults males and females, corroborating our findings. Results from multiple regression analysis in our study, showed that age, TLTM and TFM entered as the primary and most significant contributors for FNBMD in our study, accounting for between $13-21.1 \%$ and $16.2-36.9 \%$ of the explained variation in these measures in males and females, respectively. For LSBMD, TFM entered as the primary and most significant predictor accounting for $28.2 \%$ of the variation in males and $24.4 \%$ in females.

Our study is unique in several aspects; it included relatively large populations of both older adult men and women, these older people were living freely and independently among the general community, and the population had a high degree of occupational engagement. Also the homogeneity of our sample reduces ethnic, socioeconomic, nutritional and lifestyles variability, which may accentuate the more important determinants. There were, however, several limitations associated with this study. The cross-sectional design does not allow conclusions about the cause-and-effect relationship between PA and BMD. Although the Baecke questionnaire has been shown to have acceptable reproducibility $(r=0.71-0.82)$, the limited ability of some participants to accurately recall past sport and leisure activities could introduce bias and lead to misclassification. In addition, PA questionnaires are unlikely to be sufficiently sensitive to detect all the mechanical loading effects in all bone types and all bone regions. In our study, although we used the Tritrac accelerometer in a small sub-sample with similar results to the Baecke questionnaire, this analysis was also probably insufficiently sensitive to detect subtle effects of differing types of loading parameters. This issue needs to be addressed in future studies. The data were obtained from independently living older adult men and women from Madeira, Portugal, a geographically isolated region where the cultural backgrounds, living and working conditions and environmental influences are generally homogeneous. The homogeneity of these environmental influences, especially with regards to working history, would minimize the apparent importance of PA as a determinant of BMD and bone strength in this study. Further, the participants were essentially volunteers, who could have been generally healthier than those who did not participate, and survivor bias, especially among males in the older age-cohorts cannot be ruled out as a potential confounding factor particularly for between sex comparisons in our study. Lastly, ours was a very unique older population as witnessed by their lower rates of retirement, high prevalence of gainful employment in farming and reduced dependency on social assistance.

In conclusion, our findings point to the importance of age, TLTM and TFM in both sexes, as predominant determinants of bone health in older Portuguese men and women. Habitual PA showed significant positive correlations with BMD for the total body and FNBMD in women, but was either not important or relatively less important than constitutive factors in explaining variation in BMD or FSI. Neither dietary calcium intake nor alcohol consumption appeared as important determinants of bone health in this study. Our findings suggest that bone health promotion and preservation might be enhanced among the older adults by encouraging dietary practices and PA behaviors aimed at body composition stabilization with advancing age.

\section{Funding}

This work was supported by doctoral degree grants from Fundação para a Ciência e a Tecnologia reference: SFRH/BD/ $29300 / 2006$. Technical assistance in collection of the data was supported by Madeira Regional Government and Regional Secretary of Education and Culture. The dual-energy X-ray absorptiometryDXA scans were generously sponsored by Iberdata Equipment's S.A.

\section{Conflict of interest statement}

The author(s) declare that they have no personal or financial conflict of interest.

\section{Acknowledgments}

We would like to greatly acknowledge the supervision and assistance of Professor Emeritus Gaston Beunen of the Faculty of Kinesiology and Rehabilitation Sciences, Department of Biomedical Kinesiology, Katholieke Universiteit Leuven, who assisted in the data analysis and contributed significantly to the quality of the manuscript in its early stages. Gaston unexpectedly passed away in August of 2011. The authors are also grateful to Ercília Fena, Joana Castro and Letícia Sousa for technical assistance in the collection of the sample and data management. 


\section{References}

Baecke, J. A., Burema, J., \& Frijters, J. E. (1982). A short questionnaire for the measurement of habitual physical activity in epidemiological studies. The American Journal of Clinical Nutrition, 36, 936-942.

Beck, T. J., Ruff, C. B., Warden, K. E., Scott, W. W., Jr., \& Rao, G. U. (1990). Predicting femoral neck strength from bone mineral data: A structural approach. Investigative Radiology, 25, 6-18.

Booth, M. L., Owen, N., Bauman, A., Clavisi, O., \& Leslie, E. (2000). Social-cognitive and perceived environment influences associated with physical activity in older Australians. Preventive Medicine, 31, 15-22.

Daly, R. M., Ahlborg, H. G., Ringsberg, K., Gardsell, P., Sernbo, I., \& Karlsson, M. K. (2008). Association between changes in habitual physical activity and changes in bone density, muscle strength, and functional performance in elderly men and women. Journal of the American Geriatrics Society, 56, 2252-2260.

Dargent-Molina, P., Poitiers, F., Bréart, G., \& EPIDOS, Group., (2000). In elderly women weight is the best predictor of a very low bone mineral density: Evidence from the EPIDOS study. Osteoporosis International, 11, 881-888.

Dishaman, R. K., Heath, G. W., \& Lee, I. (2013). Physical activity epidemiology (2nd ed.). Champaign, IL: Human Kinetics.

Dytfeld, J., Ignaszak-Szczepaniak, M., Gowin, E., Michalak, M., \& Horst-Sikorska, W. (2011). Influence of lean and fat mass on bone mineral density (BMD) in postmenopausal women with osteoporosis. Archives of Gerontology and Geriatrics, 53, $237-242$.

Eisman, J. A. (1999). Genetics of osteoporosis. Endocrine Reviews, 20, 788-804.

Erdfelder, E., Faul, F., \& Buchner, A. (1996). GPOWER: A general power analysis program. Behavior Research Methods, 28, 1-11.

Faulkner, K. G., Wacker, W. K., Barden, H. S., Simonelli, C., Burke, P. K., Ragi, S., et al. (2006). Femur strength index predicts hip fracture independent of bone density and hip axis length. Osteoporosis International, 17, 593-599.

Felson, D. T., Zhang, Y., Hannan, M. T., \& Anderson, J. J. (1993). Effects of weight and body mass index on bone mineral density in men and women: The Framingham study. Journal of Bone and Mineral Research, 8, 567-573.

Gába, A., Kapuš, O., Pelclová, J., \& Riegerová, J. (2012). The relationship between accelerometer-determined physical activity (PA) and body composition and bone mineral density (BMD) in postmenopausal women. Archives of Gerontology and Geriatrics, 54, 315-321.

Hagberg, J. M., Zmuda, J. M., McCole, S. D., Rodgers, K. S., Ferrell, R. E., Wilund, K. R., et al. (2001). Moderate physical activity is associated with higher bone mineral density in postmenopausal women. Journal of the American Geriatrics Society, 49, 1411-1417.

Hannan, M. T., Felson, D. T., Dawson-Hughes, B., Tucker, K. L., Cupples, L. A., Wilson, P. W., et al. (2000). Risk factors for longitudinal bone loss in elderly men and women: The Framingham Osteoporosis Study. Journal of Bone and Mineral Research, 15, 710-720.

Ho-Pham, L. T., Nguyen, N. D., Lai, T. Q., \& Nguyen, T. V. (2010). Contributions of lean mass and fat mass to bone mineral density: A study in postmenopausal women. BMC Musculoskeletal Disorders, 11, 59.

International Osteoporosis Foundation (IOF). (2001). Osteoporosis in the European Community: a call to action. An audit of policy developments since 1998 Available from http://www.iofbonehealth.org/publications/eu-policy-report-of-2001.html Accessed 15.04.11.

International Osteoporosis Foundation (IOF). (2008). Osteoporosis in the European Union in 2008: Ten years of progress and ongoing challenges Available from http://www.iofbonehealth.org/publications/eu-policy-report-of-2008.html.

Jones, G., Nguyen, T., Sambrook, P., Kelly, P. J., \& Eisman, J. A. (1994). Progressive loss of bone in the femoral neck in elderly people: Longitudinal findings from the Dubbo osteoporosis epidemiology study. British Medical Journal, 309, 691-695.

Kanis, J. A., Burlet, N., Cooper, C., Delmas, P. D., Reginster, J. Y., Borgstrom, F., et al. (2008). European guidance for the diagnosis and management of osteoporosis in postmenopausal women. Osteoporosis International, 19, 399-428.

Kanis, J. A., \& Johnell, O. (2005). Requirements for DXA for the management of osteoporosis in Europe. Osteoporosis International, 16, 229-238.

Kenny, A. M., Prestwood, K. M., Marcello, K. M., \& Raisz, L. G. (2000). Determinants of bone density in healthy older men with low testosterone levels. The Journals of Gerontology. Series A, Biological Sciences and Medical Sciences, 55, 492-497.

Khan, K., McKay, H., Kannus, P., Bailey, D., Wark, J., \& Bennell, K. (2001). Physical activity and bone health. Champaign, IL: Human Kinetics.

Lane, N. E. (2006). Epidemiology, etiology, and diagnosis of osteoporosis. American Journal of Obstetrics and Gynecology, 194, S3-S11.

Lau, E. M., Leung, P. C., Kwok, T., Woo, J., Lynn, H., Orwoll, E., et al. (2006). The determinants of bone mineral density in Chinese men-results from Mr. Os (Hong Kong), the first cohort study on osteoporosis in Asian men. Osteoporosis International, 17, 97-303.

Lim, K., \& Taylor, L. (2005). Factors associated with physical activity among older people a population-based study. Preventive Medicine, 40, 33-40.

Looker, A. C., Melton, L. J., 3rd, Harris, T., Borrud, L., Shepherd, J., \& McGowan, J. (2009). Age, gender, and race/ethnic differences in total body and subregional bone density. Osteoporosis International, 20, 1141-1149.
Lopes, C. (2000). Reproducibility and validity of a semi-quantitative food-frequency questionnaire (dissertation) Porto, Portugal: University of Porto (in Portuguese).

Maïmoun, L., \& Sultan, C. (2011). Effects of physical activity on bone remodeling. Metabolism: Clinical and Experimental, 3, 373-388.

Mavroeidi, A., Stewart, A. D., Reid, D. M., \& Macdonald, H. M. (2009). Physical activity and dietary calcium interactions in bone mass in Scottish postmenopausal women. Osteoporosis International, 20, 409-416.

Nahas, E. A., Kawakami, M. S., Nahas-Neto, J., Buttros, Dde. A., Cangussu, L., \& Rodrigues, A. B. (2011). Assessment of risk factors for low bone mineral density in Brazilian postmenopausal women. Climacteric: The Journal of the International Menopause Society, 14, 220-227.

Nguyen, T. V., Center, J. R., \& Eisman, J. A. (2000). Osteoporosis in elderly men and women: effects of dietary calcium, physical activity, and body mass index. Journal of Bone and Mineral Research, 15, 322-331.

Nguyen, T. V., Sambrook, P. N., \& Eisman, J. A. (1998). Bone loss, physical activity, and weight change in elderly women: the Dubbo Osteoporosis Epidemiology Study. Journal of Bone and Mineral Research, 13, 1458-1467.

Nurzenski, M. K., Briffa, N. K., Price, R. I., Khoo, B. C., Devine, A., Beck, T. J., et al. (2007). Geometric indices of bone strength are associated with physical activity and dietary calcium intake in healthy older women. Journal of Bone and Mineral Research, 22, 416-424.

Ono, R., Hirata, S., Yamada, M., Nishiyama, T., Kurosaka, M., \& Tamura, Y. (2007). Reliability and validity of the Baecke physical activity questionnaire in adult women with hip disorders. BMC Musculoskeletal Disorders, 8, 61.

Philippaerts, R. M., \& Lefevre, J. (1998). Reliability and validity of three physical activity questionnaires in Flemish males. American Journal of Epidemiology, 147, 982-990.

Pluijm, S. M., Visser, M., Smit, J. H., Popp-Snijders, C., Roos, J. C., \& Lips, P. (2001) Determinants of bone mineral density in older men and women: Body composition as mediator. Journal of Bone and Mineral Research, 16, 2142-2151.

Reid, I. R., Plank, L. D., \& Evans, M. C. (1992). Fat mass is an important determinant of whole body bone density in premenopausal women but not in men. The Journal of Clinical Endocrinology and Metabolism, 75, 779-782.

Rose, D. J. (2003). Fallproof: A comprehensive balance and mobility training program. Champaign, IL: Human Kinetics.

Schoffl, I., Kemmler, W., Kladny, B., Vonstengel, S., Kalender, W. A., \& Engelke, K. (2008). In healthy elderly postmenopausal women variations in BMD and BMC at various skeletal sites are associated with differences in weight and lean body mass rather than by variations inhabitual physical activity, strength or VO2 max. Journal of Musculoskeletal \& Neuronal Interactions, 8, 363-374.

Semanick, L. M., Beck, T. J., Cauley, J. A., Wheeler, V. W., Patrick, A. L., Bunker, C. H., et al (2005). Association of body composition and PA with proximal femur geometry in middle-aged and elderly Afro-Caribbean men: the Tobago bone health study. Calcified Tissue International, 77, 160-166.

Statistics Portugal. (2002). Census 2001. Final results: XIV Population census. IV Housing census. Lisbon: Statistics Portugal (in Portuguese).

Statistics Portugal. (2009). Resident Population Projections - Portugal - 2008-2060. Lisbon: Statistics Portugal. Department of Social and Demographic Statistics (in Portuguese).

Stenderup, K., Justesen, J., Clausen, C., \& Kassem, M. (2003). Aging is associated with decreased maximal life span and accelerated senescence of bone marrow stromal cells. Bone, 33, 919-926.

Stewart, K. J., Bacher, A. C., Hees, P. S., Tayback, M., Ouyang, P., \& Jan de Beur, S. (2005). Exercise effects on bone mineral density relationships to changes in fitness and fatness. American Journal of Preventive Medicine, 28, 453-460.

Travison, T. G., Araujo, A. B., Esche, G. R., Beck, T. J., \& McKinlay, J. B. (2008). Lean mass and not fat mass is associated with male proximal femur strength. Journal of Bone and Mineral Research, 23, 189-198.

U.S. Department of Health and Human Services. (2004). Bone health and osteoporosis: A report of the surgeon general. Rockville, MD: U.S. Department of Health and Human Services, Office of the Surgeon General Retrieved from http://www.surgeongeneral.gov/library.

Uusi-Rasi, K., Sievänen, H., Pasanen, M., Beck, T. J., \& Kannus, P. (2008). Influence of calcium intake and PA on proximal femur bone mass and structure among pre- and postmenopausal women. A 10-year prospective study. Calcified Tissue International, 82, 171-181.

Vuillemin, A., Guillemin, F., Jouanny, P., Denis, G., \& Jeandel, C. (2001). Differential influence of PA on lumbar spine and femoral neck bone mineral density in the elderly population. The Journals of Gerontology. Series A, Biological Sciences and Medical Sciences, 56, 248-253.

Walsh, M. C., Hunter, G. R., \& Livingstone, M. B. (2006). Sarcopenia in premenopausal and postmenopausal women with osteopenia, osteoporosis and normal bone mineral density. Osteoporosis International, 17, 61-67.

Yoshikawa, T., Turner, C. H., Peacock, M., Slemenda, C. W., Weaver, C. M., Teegarden, D., et al. (1994). Geometric structure of the femoral neck measured using dualenergy X-ray absorptiometry. Journal of Bone and Mineral Research, 9, $1053-$ 1064. 